\title{
Representative democracy and the implementation of majority-preferred alternatives
}

\section{Citation}

Coffman, Katherine Baldiga. 2015. "Representative Democracy and the Implementation of Majority-Preferred Alternatives." Social Choice and Welfare 46 (3) (September 17): 477-494. doi:10.1007/s00355-015-0922-3.

\section{Published Version}

10.1007/s00355-015-0922-3

\section{Permanent link}

http://nrs.harvard.edu/urn-3:HUL.InstRepos:37221621

\section{Terms of Use}

This article was downloaded from Harvard University's DASH repository, and is made available under the terms and conditions applicable to Open Access Policy Articles, as set forth at http:// nrs.harvard.edu/urn-3:HUL.InstRepos:dash.current.terms-of-use\#OAP

\section{Share Your Story}

The Harvard community has made this article openly available.

Please share how this access benefits you. Submit a story.

\section{Accessibility}




\title{
Representative Democracy and the Implementation of Majority-preferred Alternatives
}

\author{
Katherine Baldiga Coffman* \\ The Ohio State University
}

May 18, 2015

\begin{abstract}
In this paper, we contrast direct and representative democracy. In a direct democracy, individuals have the opportunity to vote over the alternatives in every choice problem the population faces. In a representative democracy, the population commits to a candidate ex ante who will then make choices on its behalf. While direct democracy is normatively appealing, representative democracy is the far more common institution because of its practical advantages. The key question, then, is whether representative democracy succeeds in implementing the choices that the group would make under direct democracy. We find that, in general, it does not. We model a population as a distribution of voters with strict preferences over a finite set of alternatives and a candidate as a strict ordering of those alternatives that serves as a binding, contingent plan of action. We focus on the case where the direct democracy choices of the population are consistent with a strict ordering of the alternatives. We show that even in this case, where the normative recommendation of direct democracy is clearest, representative democracy may not elect the candidate with this ordering. Keywords: direct
\end{abstract}

*Mailing Address: 1945 N. High St, Arps Hall 427, Columbus, OH 43210; Email: coffman.201@osu.edu; Telephone: 614-247-8718. 
democracy, representative democracy, majoritarianism, social choice, JEL Codes: D¹, D72 


\section{Introduction}

Direct democracy is a fundamental principle of collective decision-making. When a choice problem arrives, individuals should have the opportunity to express preferences over the alternatives. A good decision-making rule then aggregates these preferences into a choice that reflects the will of the group. While many aspects of social decision-making have been debated, this individual right to direct participation has remained a normative ideal from both a theoretical and popular standpoint.

Yet, despite its normative appeal, direct democracy is relatively rare in practice. Just over half of the states in the United States allow for recalls and/or popular referendums and no forms of direct democracy exist at the federal level (NCSL.org). Direct democracy plays a similarly minor role in the governments of countries around the world, with the well-studied exception of Switzerland (Frey 1994). Most institutions instead take the form of representative democracies, under which elected officials make decisions on behalf of the electorate. From a practical perspective, representative democracies have an edge over direct democracies as they dramatically reduce transaction costs and shift the burden of decision-making to a small group of well-informed leaders. The question, then, is whether representative democracy, with its practical advantages, can successfully implement the choices that would be reached under the more normatively attractive direct democracy. ${ }^{1}$

This paper tackles this question from a theoretical perspective. We take a majoritarian approach to modeling both direct and representative democracy. In addition to being a widely-implemented and well-accepted political principle, majoritarianism has been shown to be a collective decision-making rule that works well over a large class of domains (Dasgupta and Maskin 2008). To analyze outcomes under direct democracy, we simply look at the tournament over alternatives induced by majority rule. We focus on populations where majority preferences over alternatives produce a ranking of alternatives, with each alternative

\footnotetext{
${ }^{1}$ There is a rich existing literature on representative democracy, though in general this literature has focused more on voting power and proportional representation. See, for example, Tullock (1967), Chamberlin and Courant (1983), Monroe (1995), Felsenthal and Machover (1998), and Potthof and Brams (1998).
} 
majority-preferred to all others below it in the ranking. ${ }^{2}$ In these cases, the normative recommendation for representative democracy is most clear: we expect that the candidate whose preferences best match this ordering of alternatives should be elected.

In our model of representative democracy, members of the population vote over candidates rather than alternatives. To simplify our setting, we define a candidate as an ordinal ranking of alternatives: a binding, contingent plan of action for future choice problems. We assume that when a choice problem of alternatives arrives, the social decision is made according to the ordering of the elected candidate. ${ }^{3}$ The key modeling assumption is how individuals vote over candidates. We use the Kemeny distance to map preferences over alternatives into preferences over candidates. That is, when faced with a pairwise choice between candidates, we assume that an individual votes for the candidate with whom she is most likely to agree about the choice from a randomly selected pair of alternatives. Once we know individuals' preferences over candidates, we can compare these candidates pairwise. In a comparison of two candidates, the winning candidate is the candidate who earns a majority of the population's votes: the candidate with whom the majority of the population is closer to according to the Kemeny distance. This type of political action generates a tournament over the candidates. We use the uncovered set to identify the winning candidates given an arbitrary tournament structure. The uncovered set is an appealing solution concept, as it is Condorcet consistent and contains only Pareto undominated orderings. The uncovered set also contains many other popular tournament solution concepts, which allows us to generalize our results to a variety of other settings.

We find that for problems with a small number of alternatives, representative democracy does succeed in electing candidates that implement the choices made under direct democracy. But, for general problems, we derive a negative result. We show that even for the

\footnotetext{
${ }^{2}$ This is the case where the tournament over alternatives is not only complete and asymmetric, but also transitive.

${ }^{3}$ In the language of Edward Burke, this is a delegate model of representative democracy, rather than a trustee model, as the elected official is committed to enacting the platform selected by the voters. See Maskin and Tirole (2004) or Fox and Shotts (2013) for discussions of the delegate versus trustee debate.
} 
most well-behaved populations, where majority preferences over the alternatives are consistent with a strict ordering, representative democracy may not elect the candidate with this ordering. That is, when given a chance to commit ex ante to a binding plan of action, the population may select a plan which contradicts the majority preferences on some choice problems. Following our results section, we discuss related works that highlight the more general difficulty in identifying forms of representative democracy that are consistent with direct democracy.

\section{Notation and Model}

We first lay out notation and then discuss our model.

\section{$2.1 \quad$ Notation}

We will adopt much of the notation of Baldiga and Green (2013). We use a finite space of alternatives, $X=\left\{a_{1}, a_{2}, \ldots, a_{n}\right\}$. A preference, denoted $\pi$, is a strict ordering of the alternatives, where $\pi$ corresponds to a permutation of the integers $\{1, \ldots, n\}$; given the preference $\pi=\left(a_{\pi(1)} a_{\pi(2)} \ldots a_{\pi(n)}\right), a_{i}$ is preferred to $a_{j}$ if and only if $\pi^{-1}(i)<\pi^{-1}(j)$. The set of all $n$ ! preferences over $X$ is $\Pi$. It will be useful to write $e$ to represent the natural ordering of the alternatives, $e=\left(a_{1} a_{2} \ldots a_{n}\right) \in \Pi$.

A population, $\lambda$, is a distribution over $\Pi$. Let $\Lambda$ be the set of all distributions over $\Pi$.

We model a candidate as a strict ordering of the alternatives in $X$, which serves as a binding, contingent plan of action. We will write candidate $\pi$ to denote the candidate with ordering $\pi$. In all analysis below, we consider all candidates to be available; that is, the set of candidates is equal to $\Pi$.

We model these majoritarian systems using tournaments. A tournament is a complete, asymmetric binary relation. Our analysis considers two types of tournaments: tournaments on the alternative space and tournaments on the candidate space. We use $\Gamma(X)$ to denote a 
tournament on the space of alternatives; we reserve the traditional $T$ to refer to a tournament on the space of candidates, $T(\Pi)$. In both cases, a tournament depends upon the preferences of the population; therefore, we write $\Gamma^{\lambda}$ or $T^{\lambda}$ to denote the tournaments generated by a population $\lambda$.

\subsection{Models of Direct and Representative Democracy}

Under direct democracy, members of the population vote over alternatives. We use the majority tournament to model these decisions. In this tournament, the relationship between any pair of alternatives is determined by majority rule: $a_{i} \Gamma^{\lambda} a_{j}$ if $\sum_{\pi \in \Pi}\left(\lambda\left(\pi \mid a_{i} \succ a_{j}\right)\right)>$ $\sum_{\pi \in \Pi}\left(\lambda\left(\pi \mid a_{j} \succ a_{i}\right)\right) .{ }^{4}$ We write $a_{i} \Gamma^{\lambda} a_{j}$ if $a_{i}$ beats $a_{j}$ in the tournament $\Gamma^{\lambda}(X)$. In the populations we consider below, majority rule over alternatives will be consistent with a strict ordering. We study this case because of the clear normative recommendation available. When the majority rule tournament over alternatives produces a strict ordering, then in order for representative democracy to be consistent with direct democracy, it must also produce this ordering. In other cases, when majority preferences over alternatives are not consistent with a strict ordering, there is no clear expectation for how representative democracy should behave. Therefore, we choose only to define and test for consistency in the case when majority preferences over alternatives do not cycle. ${ }^{5}$

Building a model of representative democracy requires a theory of how individuals choose to vote over candidates. We use the Kemeny distance (also known as the bubble sort distance and the Kendall distance) to define individuals' preferences over candidates. The Kemeny distance between $\pi$ and $\pi^{\prime}$, which we will denote $f\left(\pi, \pi^{\prime}\right)$, is the number of pairs of alternatives the two orderings rank differently (Kemeny 1959 and Kemeny Snell 1962); we assume that voters prefer candidates who are closer (in terms of Kemeny distance) to their own

\footnotetext{
${ }^{4}$ We restrict attention to populations with no ties in the majority relation, guaranteeing a complete and asymmetric tournament. This is similar to assuming an odd number of voters in the more common discrete population framework.

${ }^{5}$ This is consistent with the approach of Laffond and Laine (2000), who also focus on the case where majority preferences over alternatives produce an ordering.
} 
ordering of alternatives. Formally, given two candidates $\pi$ and $\pi^{\prime}$, a voter with preference $\pi^{\prime \prime}$ will prefer $\pi$ if and only if $f\left(\pi^{\prime \prime}, \pi\right)<f\left(\pi^{\prime \prime}, \pi^{\prime}\right)$. This is equivalent to assuming that a voter prefers candidate $\pi$ to candidate $\pi^{\prime}$ if she is more likely to agree with candidate $\pi$ about the ranking of a randomly-drawn pair of alternatives (Baldiga and Green (2013) formalize this interpretation). Of course, there are other ways of mapping preferences over alternatives into preferences over candidates, and our results will be sensitive to this particular choice. However, in order for our negative result to be as strong and surprising as possible, we want to create as much parallelism as we can between the direct and representative democracy models. Using the Kemeny distance to define preferences over candidates creates an appealing symmetry between the direct and representative democracy models. The preferences over candidates defined by $f\left(\pi, \pi^{\prime}\right)$ depend only on each voter's pairwise preferences over alternatives, just as the preferences over alternatives defined by majority rule do. Previous work in this area has also used the Kemeny distance to determine voters' preferences over orderings. For instance, in their investigation of the strategy-proofness of social welfare functions, Bossert and Storcken (1992) assume, as we do, that individuals' preferences over orderings are determined by relative proximity under the Kemeny distance. In Figure 1, we provide an illustration of the Kemeny distances between the six strict orderings in $\Pi$ for the $n=3$ case.

\section{Insert Figure 1 Here}

Figure 1: We use a hexagon to demonstrate the space of orderings for the $n=3$ case, with each vertex representing an ordering. Above, we illustrate the Kemeny distances from $e=a_{1} a_{2} a_{3}$ to each of the other orderings in the space.

Voters' preferences over candidates generate a tournament on $\Pi$, which we denote $T^{\lambda}$. Consider two strict orderings, $\pi^{\prime}$ and $\pi$. Define a subset of preferences, $\Pi_{1}$, such that for all $\pi^{\prime \prime} \in \Pi_{1}$, we have $f\left(\pi^{\prime \prime}, \pi^{\prime}\right)<f\left(\pi^{\prime \prime}, \pi\right)$, and define a subset of preferences, $\Pi_{2}$, such that 
for all $\pi^{\prime \prime} \in \Pi_{2}$, we have $f\left(\pi^{\prime \prime}, \pi\right)<f\left(\pi^{\prime \prime}, \pi^{\prime}\right)$. Then, we will say that $\pi^{\prime}$ defeats $\pi$, that is $\pi^{\prime} T^{\lambda} \pi$, if $\lambda\left(\Pi_{1}\right)>\lambda\left(\Pi_{2}\right)$. In the event that $\lambda\left(\Pi_{1}\right)=\lambda\left(\Pi_{2}\right)$, we can use a random tie-breaking rule such as a coin flip to determine the $T^{\lambda}$ relationship between $\pi$ and $\pi^{\prime}$. Note that in the space of distributions over strict orderings, this is a non-generic event. Furthermore, none of the results presented below depend upon this assumption. This simply assures that all tournaments are complete and asymmetric. ${ }^{6}$

\subsection{Tournament Solutions}

We consider only the simplest direct democracy tournaments, restricting our attention to those tournaments generated by populations in which majority rule over alternatives is consistent with a strict ordering. In these cases, there is a clear winner of the tournament: the alternative which defeats all others in the tournament relation, the Condorcet winner. Furthermore, because there are no cycles, we can describe the tournament by a strict ordering where each alternative is majority-preferred to all those below it.

However, for general tournaments, including the tournaments over strict orderings we study below, identifying the winners is non-trivial as the tournament relation may cycle. Therefore, we need a tournament solution that will determine the best elements given an arbitrary tournament structure. We use the uncovered set as our tournament solution (see Gilles (1959), Fishburn (1977), and Miller (1980) for early work on this topic). We follow the

\footnotetext{
${ }^{6}$ This definition means we employ relative majority rule. We could alternatively define our tournaments in terms of absolute majority rule. Under this assumption, we would have $\pi^{\prime} T^{\lambda} \pi$ only if a majority of voters are strictly closer to $\pi^{\prime}$ than $\pi$. For absolute majority rule, if neither candidate is closer to more than half of the population, the two candidates would tie in the tournament relation.

In our framework, voters will often be indifferent between candidates. Each voter has a set of indifference curves: a voter most prefers the candidate with his own ordering, then he equally prefers all candidates with whom he disagrees about the choice from one pair of alternatives, and next he equally prefers all candidates with whom he disagrees about the choice from two pairs of candidates, etc.

Given the large amount of indifference in our population, choosing to use absolute majority rule would result in a large number of ties in our tournaments over orderings. These ties would disregard the information we have on the voters who are not indifferent. For instance, in a tie between candidate $\pi$ and $\pi^{\prime}$, we may have that $30 \%$ of the voters are indifferent between candidate $\pi$ and candidate $\pi^{\prime}, 49 \%$ of the voters prefer $\pi$ to $\pi^{\prime}$, and only $21 \%$ of voters prefer $\pi^{\prime}$ to $\pi$. Despite the large disparity in the number of voters that strictly prefer $\pi$ to $\pi^{\prime}$ as opposed to $\pi^{\prime}$ to $\pi$, these two orderings would tie in the tournament relation. By using relative majority rule, we use this information on strict preference, even in the cases where large subsets of voters are indifferent between the two candidates.
} 
definition given by Laslier (1997), applied to our tournaments over strict orderings. First, define the covering relation of $T$. For a given $T$, we say $\pi_{i}$ covers $\pi_{j}$ if and only if:

(a) $\pi_{i} T \pi_{j}$, and

(b) for all $\pi_{k} \in \Pi, \pi_{j} T \pi_{k} \Rightarrow \pi_{i} T \pi_{k}$

The uncovered set of $T$ is the set of maximal elements of the covering relation: $\pi_{i} \in$ $U C(T)$ if and only if there does not exist $\pi_{j} \in \Pi$ such that $\pi_{j}$ covers $\pi_{i}$.

As Miller (1980) describes, the uncovered set has a number of appealing properties. The uncovered set is always a non-empty subset of the top cycle. And, unlike the top cycle, it contains only Pareto undominated orderings. ${ }^{7}$ It is Condorcet consistent: if a Condorcet winner exists, it will be the sole member of the uncovered set. The uncovered set also characterizes the outcomes under a variety of familiar voting rules. Miller (1980) and Shepsle and Weingast (1984) have shown that a number of voting procedures under both sincere and sophisticated voting implement elements of the uncovered set. These results motivate our use of the uncovered set. By working with this tournament solution, we avoid making specific institutional assumptions. Instead, we identify the likely winners under majoritarian voting rules more generally, under both sincere and strategic voting. Most importantly, for our purposes, the uncovered set contains many other familiar tournament solution concepts. Thus, the negative result we prove in this framework extends to a wide variety of other reasonable approaches.

With this solution concept in place, we are ready to define consistency between direct and representative democracy in our setting.

Definition 1 If majority preferences over alternatives are consistent with a strict ordering, $\pi$, and $\pi \in U C\left(T^{\lambda}\right)$, we will say that order consistency holds. If, in addition, $U C\left(T^{\lambda}\right)$ is a singleton, we will say that strong order consistency holds.

Note that $U C\left(T^{\lambda}\right)$ will be a singleton if and only if there is a Condorcet winner of this

\footnotetext{
${ }^{7}$ Note that a positive result (consistency between these forms of direct and representative democracy) could be derived if one chose to use the less selective top cycle as the tournament solution concept. We discuss this in more detail in Section 3.
} 
tournament. Thus, strong order consistency requires that the candidate consistent with majority preferences is selected as the unique Condorcet winner of the tournament over candidates. In the analysis that follows, we show that strong order consistency holds for the case of $n=3$ but fails for problems with $n>3$. Then, we provide a counterexample that illustrates order consistency fails for $n \geqq 10$.

\section{Consistency Results for Direct and Representative Democracy}

First, we introduce a useful proposition.

Proposition 1 Consider a population where majority preferences are consistent with $e$. Consider a strict ordering $\pi$. Obtain $\pi^{\prime}$ from $\pi$ by transposing exactly one pair of adjacent alternatives that appear in the natural order in $\pi$ (that is, transpose a pair of adjacent alternatives that $\pi$ ordered in line with majority preference). Then, it must be that $\pi T^{\lambda} \pi^{\prime}$

for all such $\pi^{\prime}$. Furthermore, if we take any of these $\pi^{\prime}$ and obtain $\pi^{\prime \prime}$ from $\pi^{\prime}$ by transposing exactly one pair of adjacent alternatives that appeared in the natural order in both $\pi$ and $\pi^{\prime}$, we must have $\pi T^{\lambda} \pi^{\prime \prime}$.

Proof: Intuitively, when $\pi$ and $\pi^{\prime}$ agree on all but a single pair of alternatives, a voter with the ordering $\pi^{\prime \prime \prime}$ will be closest to whichever of these orderings it agrees with on the pair in the question. Since $\pi$ agrees with the majority preference on the pair in question, a greater share of the population must be closer to $\pi$, yielding $\pi T^{\lambda} \pi^{\prime}$.

We can take this logic one step further to prove the claim for cases where $\pi$ and $\pi^{\prime \prime}$ agree on all but two pairs of alternatives. Denote by $\alpha$ the subset of the population that agrees with $\pi$ on the first pair in question (the one transposed to obtain $\pi^{\prime}$ ). Denote by $\beta$ the subset of the population that agrees with $\pi$ on the second pair in question (the one transposed to obtain $\pi^{\prime \prime}$ ). Because $\pi$ is consistent with the majority preference on both of these pairs, we 
know that $\lambda(\alpha)>\frac{1}{2}$ and $\lambda(\beta)>\frac{1}{2}$. Therefore:

$$
2 \times(\lambda(\alpha \cap \beta))+\lambda(\alpha \backslash \beta)+\lambda(\beta \backslash \alpha)>1
$$

We can rearrange this expression to show that:

$$
\lambda(\alpha \cap \beta)>1-(\lambda(\alpha \backslash \beta)+\lambda(\beta \backslash \alpha)+\lambda(\alpha \cap \beta))
$$

The left-hand side of this equation is the fraction of the population that agrees with $\pi$ on both of the pairs in question and will be closer to $\pi$. The right-hand side of this equation is the fraction of the population that disagrees with $\pi$ on both of the pairs in question and will be closer to $\pi^{\prime \prime}$. The rest of the population will be equidistant. Thus, the equation tells us that a larger fraction of the population will be closer to $\pi$ than $\pi^{\prime \prime}$, yielding $\pi T^{\lambda} \pi^{\prime \prime}$.

The following corollary is a straightforward implication of Proposition 1 and will prove useful in the following sections.

Corollary 1 A strict ordering that is consistent with the majority preferences of a population $\lambda$, call this $\pi^{*}$, must beat all strict orderings that are no more than two transpositions away from it; that is, we must have $\pi^{*} T^{\lambda} \pi$ for all $\pi$ such that $f\left(\pi^{*}, \pi\right) \leq 2$.

\subsection{Consistency for $n=3$}

We exploit this knowledge of the structure of tournaments over orderings to prove results for the $n=3$ case for populations with majority preferences consistent with a strict ordering.

Proposition 2 For $n=3, U C\left(T^{\lambda}\right)$ satisfies strong order consistency.

Proof: We will work through the case where $a_{1} \Gamma^{\lambda} a_{2}, a_{2} \Gamma^{\lambda} a_{3}$, and $a_{1} \Gamma^{\lambda} a_{3}$. As Figure 1 clearly demonstrates, we know that $a_{1} a_{3} a_{2}, a_{2} a_{1} a_{3}, a_{2} a_{3} a_{1}$, and $a_{3} a_{1} a_{2}$ are each less than or equal to distance 2 from $a_{1} a_{2} a_{3}$. Therefore, by Proposition 1 , we know $a_{1} a_{2} a_{3} T^{\lambda} \pi$ for each $\pi \in\left\{a_{1} a_{3} a_{2}, a_{2} a_{1} a_{3}, a_{2} a_{3} a_{1}, a_{3} a_{1} a_{2}\right\}$. Therefore, we just need to show that $a_{1} a_{2} a_{3} T^{\lambda} a_{3} a_{2} a_{1}$ 
in order to prove that $a_{1} a_{2} a_{3}$ is the Condorcet winner of the tournament, and hence the only member of the uncovered set. We can use the fact that $a_{1} \Gamma^{\lambda} a_{3}$.

$$
\begin{gathered}
\sum_{\pi_{i} \in \Pi}\left(\lambda\left(\pi_{i}\right) \mid a_{1} \succ_{\pi_{i}} a_{3}\right)>\sum_{\pi_{i} \in \Pi}\left(\lambda\left(\pi_{i}\right) \mid a_{3} \succ_{\pi_{i}} a_{1}\right) \\
\lambda\left(a_{1} a_{2} a_{3}\right)+\lambda\left(a_{1} a_{3} a_{2}\right)+\lambda\left(a_{2} a_{1} a_{3}\right)>\lambda\left(a_{2} a_{3} a_{1}\right)+\lambda\left(a_{3} a_{1} a_{2}\right)+\lambda\left(a_{3} a_{2} a_{1}\right)
\end{gathered}
$$

This inequality states that the fraction of the population with orderings closer to $a_{1} a_{2} a_{3}$ than $a_{3} a_{2} a_{1}$ (the left-hand side) is greater than the fraction of the population with orderings closer to $a_{3} a_{2} a_{1}$ than $a_{1} a_{2} a_{3}$. Thus, $a_{1} a_{2} a_{3} T^{\lambda} a_{3} a_{2} a_{1}$, and $U C\left(T^{\lambda}\right)=\left\{a_{1} a_{2} a_{3}\right\}$.

\subsection{Inconsistency for General Problems}

First, we present an example that shows that for $n=4$, strong order consistency fails. Consider the following population:

\begin{tabular}{cc}
\hline$\pi$ & $\lambda(\pi)$ \\
\hline \hline$a_{1} a_{2} a_{3} a_{4}$ & .399 \\
\hline$a_{2} a_{4} a_{1} a_{3}$ & .2 \\
\hline$a_{1} a_{4} a_{3} a_{2}$ & .2 \\
\hline$a_{3} a_{4} a_{1} a_{2}$ & .201 \\
\hline
\end{tabular}

The majority preferences of this population are consistent with $e=a_{1} a_{2} a_{3} a_{4}$. But, there is no Condorcet winner of the tournament over candidates. Consider $\hat{\pi}=a_{4} a_{1} a_{3} a_{2}$. We have $f\left(\hat{\pi}, a_{1} a_{4} a_{3} a_{2}\right)<f\left(e, a_{1} a_{4} a_{3} a_{2}\right)$ and $f\left(\hat{\pi}, a_{3} a_{4} a_{1} a_{2}\right)<f\left(e, a_{3} a_{4} a_{1} a_{2}\right)$, and we have $f\left(\hat{\pi}, a_{2} a_{4} a_{1} a_{3}\right)=f\left(e, a_{2} a_{4} a_{1} a_{3}\right)$. So,

$$
\sum_{\pi \in \Pi}[\lambda(\pi) \mid f(\hat{\pi}, \pi)<f(e, \pi)]>\sum_{\pi \in \Pi}[\lambda(\pi) \mid f(e, \pi)<f(\hat{\pi}, \pi)]
$$

As a result, $\hat{\pi} T^{\lambda} e$. And, in fact, we can show that $\hat{\pi} \in U C\left(T^{\lambda}\right)$, with $U C\left(T^{\lambda}\right)=$ $\left\{e, a_{1} a_{2} a_{4} a_{3}, a_{1} a_{4} a_{2} a_{3}, \hat{\pi}\right\}$. We can extend this $n=4$ example to a problem with an arbitrary 
number of alternatives by preserving the structure above for the first four alternatives and simply appending additional alternatives in their natural order to the right end of each of the four orderings above. This leads to the following proposition.

Proposition 3 For $n>3, U C\left(T^{\lambda}\right)$ fails strong order consistency.

This is the strongest result that we can achieve for $n=4$. In the Appendix, we prove that order consistency must hold for $n=4$ populations. For $n \geq 10$, we are able to prove a stronger result. We identify populations in which the strict ordering consistent with the majority preferences is covered.

Theorem 1 For $n \geq 10, U C\left(T^{\lambda}\right)$ fails order consistency.

Proof: We prove this through a general counterexample. First, we construct the population. The majority preferences of our population $\lambda$ will be consistent with $e$ on all possible pairs drawn from among the $n$ alternatives. Select a set of $j$ pairs of alternatives, where $5 \leq j \leq \frac{n}{2}$. Each pair should consist of two adjacent elements in the natural ordering, and all pairs in the set should be disjoint. For example, it would be permissible to select $\left\{a_{1}, a_{2}\right\}$ and $\left\{a_{3}, a_{4}\right\}$ as two of the pairs, but one could not select $\left\{a_{1}, a_{2}\right\}$ and $\left\{a_{2}, a_{3}\right\}$, or $\left\{a_{1}, a_{3}\right\}$ and $\left\{a_{4}, a_{6}\right\}$. It will be helpful to have notation for the $j$ pairs; let them be denoted $p_{1}, p_{2}, \ldots, p_{j}$. Note that since the pairs consist of adjacent and disjoint alternatives, it is always possible to find a strict ordering $\pi$ that agrees with the majority preference on any particular subset of the pairs $\left\{p_{1}, p_{2}, \ldots, p_{j}\right\}$ exactly.

We will associate with each of the $j$ pairs a particular strict ordering, $\pi_{p_{i}}$, where $\pi_{p_{i}}$ agrees with the majority preference on pair $p_{i}$, disagrees with the majority preferences on the other $j-1$ pairs in the set $\left\{p_{1}, p_{2}, \ldots, p_{j}\right\}$, and agrees with the majority preference on all other pairs of alternatives.

Allocate the population as follows. Let $\lambda(e)=\frac{1}{2}-\varepsilon$, where $\varepsilon<\frac{1}{2 j}$. Divide the rest of the population evenly among the strict orderings $\left\{\pi_{p_{1}}, \pi_{p_{2}}, \ldots, \pi_{p_{j}}\right\}$, creating $j$ equal masses of size $\frac{\frac{1}{2}+\varepsilon}{j}$. 
It is straightforward to check that this population produces majority rule over alternatives that agrees with $e$. For each pair not included in $\left\{p_{1}, p_{2}, \ldots, p_{j}\right\}$, the population unanimously prefers $a_{i}$ to $a_{i+k}$, where $i, k \in\{1, . ., n-1\}$. For each of the pairs in $\left\{p_{1}, p_{2}, \ldots, p_{j}\right\}$, we have that $\left(\frac{1}{2}-\varepsilon\right)+\left(\frac{\frac{1}{2}+\varepsilon}{j}\right)>\frac{1}{2}$ agrees with $e$.

Now we will show that for this population $e \notin U C\left(T^{\lambda}\right)$. We do so by identifying a strict ordering that covers $e$. Consider the strict ordering that disagrees with the majority preferences on all of the pairs $\left\{p_{1}, p_{2}, \ldots, p_{j}\right\}$ and agrees with the majority preference on all other pairs. Denote this ordering $\hat{\pi}$. Since we are working with $f\left(\pi, \pi^{\prime}\right)$, the distance between any two orderings is, up to a scale factor, the number of pairs over which the two orderings disagree. For simplicity, we will scale our distances below to the number of pairwise disagreements.

First we will show that $\hat{\pi} T e$. We have $f\left(\pi_{p_{i}}, \hat{\pi}\right)=1$ and $f\left(\pi_{p_{i}}, e\right)=j-1 \forall i \in\{1, \ldots, j\}$. Therefore, we have $\frac{1}{2}+\varepsilon$ of the population that is closer to $\hat{\pi}$ than $e$, so $\hat{\pi} T e$.

In order to prove that $\hat{\pi}$ covers $e$, we must show that there cannot exist a $\pi^{\prime}$ such that $e T \pi^{\prime}$ but $\pi^{\prime} T \hat{\pi}$. Suppose there did exist such a $\pi^{\prime}$.

We have that $e T \pi^{\prime}$. This implies that we have $f\left(e, \pi_{p_{i}}\right) \leq f\left(\pi^{\prime}, \pi_{p_{i}}\right)$ for at least some $i \in\{1, \ldots, j\}$. Because we know $f\left(e, \pi_{p_{i}}\right)=j-1$, this implies that $f\left(\pi^{\prime}, \pi_{p_{i}}\right) \geq j-1$ for at least some $i \in\{1, \ldots, j\}$.

We also know that $\pi^{\prime} T \hat{\pi}$. Then we must have that $f\left(\pi^{\prime}, \pi_{p_{k}}\right) \leq f\left(\hat{\pi}, \pi_{p_{k}}\right)$ for at least some $k \in\{1, \ldots, j\}$. We know that $f\left(\hat{\pi}, \pi_{p_{k}}\right)=1$ for all $k \in\{1, \ldots, j\}$, which implies that $f\left(\pi^{\prime}, \pi_{p_{k}}\right) \leq 1$ for at least some $k \in\{1, \ldots, j\}$.

Finally, we know that $f\left(\pi_{p_{i}}, \pi_{p_{k}}\right) \leq 2$ for any $i, k \in\{1, \ldots, j\}$.

This creates the following violation of the triangle inequality: $f\left(\pi^{\prime}, \pi_{p_{k}}\right) \leq 1, f\left(\pi_{p_{i}}, \pi_{p_{k}}\right) \leq$ 2 , and $f\left(\pi^{\prime}, \pi_{p_{i}}\right) \geq j-1$, where $j \geq 5$. This is a contradiction.

Therefore, there can exist no $\pi^{\prime}$ such that $e T \pi^{\prime}$ but $\pi^{\prime} T \hat{\pi}$. As a result, we can conclude that $\hat{\pi}$ covers $e$. Thus, $e$ cannot be a member of the uncovered set. 
This proof describes a method for constructing populations for which direct and representative democracy yield different choices. These populations have a rather intuitive interpretation. Let us think about a population constructed by the method above for the case of $n=10$. First, we note the distinction between the five "contested" issues $\left(\left\{a_{1}, a_{2}\right\}\right.$, $\left.\left\{a_{3}, a_{4}\right\},\left\{a_{5}, a_{6}\right\},\left\{a_{7}, a_{8}\right\},\left\{a_{9}, a_{10}\right\}\right)$ and the other 40 pairwise choices that are decided unanimously. The largest mass of voters, just under half of them, have the preference $e$. Let us call these our "mainstream" voters. The remaining voters are divided evenly among five smaller minority preferences. Each minority agrees with the mainstream preference on just one of the contested issues; on the other hand, each minority group agrees with every other minority group on three of the five contested issues. In this way, the minority preferences are all more similar to one another than to the mainstream voters.

When voting directly over the alternatives, the population implements choices consistent with $e$. Most of the choices are unanimous; and, for the five contested pairs, the mainstream voters and one of the minority groups form a majority. Though the minority groups have similar preferences, when voting issue-by-issue, they never vote all together on a contested issue. As a result, the mainstream voters are able to implement their preferred choices. We can contrast this with the dynamic under representative democracy. In this setting, candidate $e$ cannot be elected. Though $e$ attracts the mainstream voters, there exist candidates that all five minority groups prefer to $e$. Consider candidate $\hat{\pi}$, who agrees with the unanimous choices of the population but disagrees with the majority preference on the five contested pairs. All five minority grouips prefer $\hat{\pi}$ to $e$; together, they consist of a majority of the population and can succeed in electing this compromise candidate. The choices of the selected candidates in representative democracy are more closely aligned with the minority preferences than the choices under direct democracy. The ability of the minority groups to compromise under representative democracy produces choices that are different than those implemented under direct democracy but that are preferred by more than half the population. 
Table 1: Summary of Consistency Results

\begin{tabular}{ccc}
\hline & Strong Order Consistency & Order Consistency \\
\hline \hline$n=3$ & + & + \\
$n=4$ & - & + \\
$5 \leq n \leq 9$ & - & $?$ \\
$n>10$ & - & - \\
\hline
\end{tabular}

+ indicates a positive result for given $\mathrm{n}$ and property; - indicates a negative result

We summarize our results in Table 1, with + indicating a positive result and - indicating a negative result. Order consistency results for $5 \leq n \leq 9$ remain an open question. Our strategy here focuses on generating populations by manipulating preferences over disjoint, adjacent pairs. This approach has been fruitful in constructing populations for which order consistency fails, though it may require more alternatives than might be necessary with other approaches.

\section{Related Work and Discussion}

Our result is closely related to the work of Laffond and Lainé (2000). Laffond and Lainé (2000) define a tournament consistency axiom that is very similar to our strong order consistency property. Their tournament consistency requires that if majority preferences over alternatives are consistent with an ordering, then the candidate with that ordering must be the Condorcet winner of the tournament over candidates. They characterize the set of preference extension rules (mappings from preferences over alternatives into preferences over candidates) that satisfy tournament consistency. There are technical differences between the two environments, including the impotant distinction that Laffond and Lainé (2000) require this type of tournament consistency over any subset of alternatives; that is, if majority preferences over some subset of alternatives are consistent with an ordering, the candidate (defined as an ordering over that subset) whose ordering is consistent with those preferences must be 
the Condorcet winner of the tournament of orderings restricted to that subset. ${ }^{8}$ But, the more important conceptual difference is that we prove that for one particular and intuitive way of defining preferences over candidates from preferences over alternatives, something even weaker than strong order consistency (or tournament consistency) fails. Not only is the candidate whose ordering is consistent with majority preferences not the Condorcet winner of the tournament (what we call a failure of strong order consistency), that candidate may not even be a member of the uncovered set of the tournament (a failure of order consistency).

Also related is the work of Lainé, Ozkes, and Sanver (working paper). In their framework, preferences over alternatives are mapped into preferences over candidates (or hyperpreferences) by a betweenness criterion: an individual with preference over alternatives $\pi$ prefers candidate $\pi^{\prime}$ to candidate $\pi^{\prime \prime}$ if the set of pairs that $\pi$ and $\pi^{\prime \prime}$ agree on is a subset of the set of pairs $\pi$ and $\pi^{\prime}$ agree on. As they describe, the preference extension defined by the Kemeny distance is a member of this family. They then define Kemeny-stability, a type of consistency property for a social welfare function. They describe a social welfare function as Kemeny-stable if a linear extension of the weak order selected by the social welfare function when applied to the population of preferences over alternatives is also selected by the social welfare function when applied to the population of preferences over candidates (where preferences over candidates are determined by the Kemeny distance). They show that many popular social welfare functions are not Kemeny stable, including unanimous scoring rules, the Copeland solution, the Slater solution, and the Kemeny rule. Their Kemeny-stability consistency property is very close to our notion of order consistency. In relation to their results, our paper could be interpreted as showing that the uncovered set fails a property similar to Kemeny-stability.

While our model is quite different, our results echo the findings of Besley and Coate

\footnotetext{
${ }^{8}$ The other technical difference is that Laffond and Laine (2000) restrict the set of preference extension rules (mappings from preferences over alternatives into preferences over candidates) they consider to those rules that produce complete linear orders over candidates, explicitly disallowing indifference. We map preferences over alternatives into preferences over candidates using the Kemeny distance, and we allow voters to be indifferent over candidates whose orderings are equidistant from their own. In this way, the particular mapping we study is not in their permissible class of preference extension rules.
} 
(2008), who study the question of whether citizens' initiatives that allow citizens to cast votes directly over issues improve upon the outcomes reached under electoral competition among representatives. Their model consists of a two party political system, where the population makes decisions in a two-dimensional policy space. Using this framework, they show that the elected candidates may implement policies that are at odds with the majority preferences of the population. They attribute these errors to the bundling of issues that is inherent in the election of a representative. As in our model, when issues are decided upon concurrently, via the choice of a representative, decisions may diverge from those made when citizens are able to vote directly over issues, one at a time. Ahn and Oliveros (2012) prove a similar result in the case where individuals' preferences over issues are not separable. They work in a game-theoretic, cardinal environment, in which voters' preferences over bundles of issues are determined by expected utility-maximization. They show that when voters' preferences over the issues are not separable, then the voting strategies that result in the election of the Condorcet winning bundle may not be an equilibrium of the model. Our paper shows that, in a much simpler, ordinal environment, even in the case where there are no complementarities or substitutabilities across issues, the bundling of choices may be distortionary.

We have chosen to use the uncovered set as the solution concept for our tournaments. How heavily does our result depend on this choice? One of the most attractive features of the uncovered set is that many other popular tournament solution concepts are subsets of the uncovered set (Laslier 1997). Therefore, it is possible to extend our negative result to many other solution concepts. This includes the basic refinements of the uncovered set, the iterated uncovered set and the minimal covering set. It also includes the Banks solution and the Bipartisan set (Banks 1985, Laffond, Laslier, and Le Breton 1993) . Another well-studied method for identifying tournament winners is ranking the members of the tournament based upon their victories and losses within the tournament. The most popular of these ranking methods include the Copeland solution, the Markov solution, and the Slater solution, each 
of which is also a refinement of the uncovered set (see, Copeland 1951, Daniels 1959, and Slater 1961). In addition, Brandt, Geist, and Harrenstein (working paper) have shown that any Pareto optimal method for selecting winners of the majoritarian tournament must be a refinement of the uncovered set; thus, our negative result extends to every rule in that family of tournament solutions as well. Thus, the comparably large size of the uncovered set is quite an attractive property for our purposes. Furthermore, the uncovered set has the appealing property that many models of strategic voting, not just sincere voting, lead to selection of members of the uncovered set; for instance, Miller (1980) develops a model of two-party electoral competition with strategic voting that leads to election of a member of the uncovered set, and Shepsle and Weingast (1984) show that the amendment procedure under strategic voting also elects a member of the uncovered set. Therefore, our negative result would also extend to these specific models of sophisticated voting.

However, if we expanded our solution concept to the top cycle (Schwartz 1972), which contains the uncovered set, we could prove a positive result. ${ }^{9}$

Proposition 4 For any population with majority preferences consistent with an ordering, $\pi^{*}, \pi^{*}$ is a member of the top cycle of the tournament over orderings.

We can prove this using Proposition 1. Start with $\pi^{*}$. By performing one transposition at a time, each time transposing a pair of alternatives that were ordered according to the majority preference, we can construct a chain, $\pi^{*} T^{\lambda} \pi_{i} T^{\lambda} \pi_{j} T^{\lambda} \ldots T^{\lambda} \pi$, from $\pi^{*}$ to any other ordering $\pi$. Thus, we must have $\pi^{*} \in T C\left(T^{\lambda}\right)$.

But for populations like the one in our counterexample, the top cycle is large; it will contain many other orderings, including our $\hat{\pi}$. Thus, while order consistency would hold, strong order consistency would fail: a candidate whose preferences are misaligned with the majority preferences over at least some issues would be electable. The results of Laffond

\footnotetext{
${ }^{9}$ Lainé, Ozkes, and Sanver (Working paper) prove a stronger version of this proposition, showing that the top cycle not only fails Kemeny-stability (similar in spirit to what we show here), but also fails their more general hyper-stability property. See Theorem 6 in their paper.
} 
and Lainé (2000) and Lainé , Ozkes, and Sanver (working paper) also highlight the more general difficulty of identifying forms of representative democracy that respect majority preferences over alternatives. Laffond and Lainé (2000) show that only lexicographic preference extension rules (preference extension rules for mapping preferences over alternatives into preferences), result in consistency between direct and representative democracy in their environment; Lainé, Ozkes, and Sanver (working paper) show that when preferences over candidates are defined by the Kemeny distance, many other familiar social welfare functions, including unanimous scoring rules, result in inconsistencies between choices under direct and representative democracies.

Our models operate on populations of strict preferences, however, the negative result that we have identified can be derived in a more basic setting as well. Consider a population of individuals who each had a yes/no preference on five different spending projects. We can apply our same rule for mapping individuals' preferences over alternatives into preferences over candidates. When deciding between two candidates, we assume that an individual votes for the candidate with whom she agrees on the greatest number of issues. In a population similar to that described in the proof of Theorem 1, the majority preference would be to vote yes on each of these five issues separately, but under representative democracy, the population would never elect the candidate who would vote yes on all five issues.

This example shows that our result does not depend on our assumption that voters' preferences take the form of orderings. What is important for our result is the existence of multiple issues. Under a direct democracy, voters express preferences over issues one at a time. Therefore, voters who may be very similar with respect to their decisions over the entire set of issues - they would agree most of the time - can end up voting differently on any one particular issue. But in a representative democracy, voters are able to express preferences over the entire set of issues by voting for a candidate. In this way, representative democracy can serve to unite and empower groups of voters with similar, but not identical preferences. 


\section{Appendix}

\subsection{Proof for $n=4$}

In this section, we prove Proposition 3 stated in Section 3.2. For $n=4, U C\left(T^{\lambda}\right)$ satisfies order consistency.

Proof: Assume majority preferences are consistent with $e$. We show that there is no ordering that can cover $e$. The key step is to recognize that we can apply Proposition 1 to rule out any ordering fewer than 5 transpositions from $e$ : $e T^{\lambda} \pi$ for any $\pi$ within two transpositions, so they cannot cover $e$, and for those three or four transpositions away, even if they beat $e$, they will be defeated by at least one ordering one or two transpositions from $e$ (which $e$ beats). So, the only orderings that could potentially cover $e$ are five or six transpositions away from $e:\left\{a_{4} a_{3} a_{1} a_{2}, a_{4} a_{2} a_{3} a_{1}, a_{3} a_{4} a_{2} a_{1}, a_{4} a_{3} a_{2} a_{1}\right\}$. We rule these out one at a time:

- We cannot have $a_{4} a_{3} a_{1} a_{2}$ covers $e$, since $e T^{\lambda} a_{2} a_{3} a_{1} a_{4} T^{\lambda} a_{4} a_{3} a_{1} a_{2}$ for any population with majority preferences consistent with $e$. Proposition 1 proves $e T^{\lambda} a_{2} a_{3} a_{1} a_{4}$. We cannot have $a_{4} a_{3} a_{1} a_{2} T^{\lambda} a_{2} a_{3} a_{1} a_{4}$ since all of the orderings closer or equidistant to $a_{4} a_{3} a_{1} a_{2}$ than $a_{2} a_{3} a_{1} a_{4}$ have $a_{4}$ precedes $a_{2}$. Thus, if more than half the population were closer to or equidistant to $a_{4} a_{3} a_{1} a_{2}$, we would not have $a_{2} \succ a_{4}$ in the majority preference.

- We cannot have $a_{4} a_{2} a_{3} a_{1}$ covers $e$, since we must have $e T^{\lambda} a_{4} a_{2} a_{3} a_{1}$. All of the orderings closer or equidistant to $a_{4} a_{3} a_{1} a_{2}$ than $e$ have $a_{4}$ precedes $a_{1}$. Thus, if more than half the population were closer to or equidistant to $a_{4} a_{2} a_{3} a_{1}$ than to $e$, we would not have $a_{1} \succ a_{4}$ in the majority preference.

- We cannot have $a_{3} a_{4} a_{2} a_{1}$ covers $e$, since $e T^{\lambda} a_{1} a_{4} a_{2} a_{3} T^{\lambda} a_{3} a_{4} a_{2} a_{1}$ for any population with majority preferences consistent with e.Proposition 1 proves $e T^{\lambda} a_{1} a_{4} a_{2} a_{3}$. We cannot have $a_{3} a_{4} a_{2} a_{1} T^{\lambda} a_{1} a_{4} a_{2} a_{3}$ since all of the orderings closer or equidistant to $a_{1} a_{4} a_{2} a_{3}$ than $a_{3} a_{4} a_{2} a_{1}$ have $a_{3}$ precedes $a_{1}$. Thus, if more than half the population were closer to or equidistant to $a_{3} a_{4} a_{2} a_{1}$, we would not have $a_{1} \succ a_{3}$ in the majority preference. 
- We cannot have $a_{4} a_{3} a_{2} a_{1}$ covers $e$, since $e T^{\lambda} a_{1} a_{3} a_{2} a_{4} T^{\lambda} a_{4} a_{3} a_{2} a_{1}$ for any population with majority preferences consistent with $e$. Proposition 1 proves $e T^{\lambda} a_{1} a_{3} a_{2} a_{4}$. We cannot have $a_{4} a_{3} a_{2} a_{1} T^{\lambda} a_{1} a_{3} a_{2} a_{4}$ since all of the orderings closer or equidistant to $a_{4} a_{3} a_{2} a_{1}$ than $a_{1} a_{3} a_{2} a_{4}$ have $a_{4}$ precedes $a_{1}$. Thus, if more than half the population were closer to or equidistant to $a_{4} a_{3} a_{2} a_{1}$, we would not have $a_{1} \succ a_{4}$ in the majority preference.

\subsection{Population Restrictions}

A natural question to ask in this context is whether we can impose restrictions on the distribution of preferences that would guarantee order consistency. This domain restriction approach has been adopted by many social choice theorists in attempts to rule out other paradoxical outcomes; perhaps most classic is the single-peakedness restriction pioneered independently by Black (1948) and Arrow (1951) and generalized by Sen and Pattanaik (1969). Their goal was to describe a class of populations for which majority rule over alternatives would not cycle. The domain restriction they proposed requires populations to be unimodal in the sense that all members of the population, for any particular triple of alternatives, must be able to agree on an alternative that is not worst. Assuming the number of voters is odd, this condition is sufficient for transitive majority rule.

Clearly, this restriction will not be enough to assure order consistency, as the class of populations we consider in our counterexample above are indeed single-peaked in terms of preferences over alternatives. However, we can use a similar idea, that of restricting the number of modes in the distribution, in order to derive a sufficient condition for order consistency in our framework. The class of populations with transitive majority rule consistent with the ordering $e$ can be thought of as having a "peak" or cluster of weight around $e$. Our sufficiency condition says that as we move away from $e$, we must not encounter another cluster of orderings similar to one another. In order to state this condition more formally, it will be useful to introduce some new terminology. When referring to a population with transitive majority rule consistent with $e$, we will call any pairwise disagreement with $e$ an 
error. For example, we will say that an ordering $\pi$ that is $m$ transpositions from $e$ contains $m$ errors. We can state our sufficiency condition in terms of these errors.

Proposition 5 Consider the class of populations with transitive majority rule consistent with e. Then, order consistency holds if for any set of $m$ errors, $m \geqq 5$, we have

$$
\sum \lambda\left(\pi \mid \pi \text { contains at least } \frac{1}{2} \text { of these } m \text { errors }\right)<\frac{1}{2}
$$

Proof. Suppose $e \notin U C\left(T^{\lambda}\right)$. We will show there must exist a set of $m$ errors, $m \geqq 5$, such that $\sum \lambda\left(\pi \mid \pi\right.$ contains at least $\frac{1}{2}$ of these $m$ errors $)>\frac{1}{2}$. Since $e \notin U C\left(T^{\lambda}\right)$, we know there exists $\hat{\pi}$ such that $\hat{\pi}$ covers $e$. Let $\hat{\pi}$ contain $m$ errors; we know $m \geqq 5$ in order for $\hat{\pi}$ to cover $e$. Since $\hat{\pi}$ and $e$ agree on all pairwise choices outside of the $m$ errors, we know that $f(\pi, \hat{\pi})$ and $f(\pi, e)$ are determined only by how many of the $m$ errors $\pi$ contains. Those $\pi$ that have less than $\frac{1}{2}$ of the $m$ errors have $f(\pi, e)<f(\pi, \hat{\pi})$. So, suppose the set of orderings that had at least $\frac{1}{2}$ of these $m$ errors in common with $\hat{\pi}$ had mass less than $\frac{1}{2}$. Then, we would have $\sum \lambda\left(\pi \mid f(\pi, e)<f(\pi, \hat{\pi})>\frac{1}{2}\right.$. This would imply $e T^{\lambda} \hat{\pi}$, contradicting $\hat{\pi}$ covers $e$.

This sufficiency condition has a straightforward intuition. If we encounter a population that contains a mass of orderings that are both (a) relatively distant from $e$, and (b) relatively close to one another, then we may have the type of counterexample presented above. This type of cluster of similar orderings far from $e$ may be able to agree upon a compromise candidate which covers $e$, but only if together they constitute a majority. The condition rules out this possibility by assuring that no such cluster of mass greater than $\frac{1}{2}$ exists.

This is a sufficient but not necessary condition for order consistency. A gray area exists between the class of populations described in our counterexample above and the class of populations described by this sufficiency condition. For some populations that fail the condition above, the distribution of mass on orderings far from $e$ may be too dispersed to agree upon an ordering like $\hat{\pi}$ which could beat everything that $e$ beats. One might ask whether 
we could improve the sufficiency condition by restricting this set of distant orderings to fall within a certain radius of one another. Below, we provide an example that illustrates why this strategy fails.

Example 1 Why Tightening the Sufficiency Condition by Restricting the Radius of the Outlier Orderings Does Not Work

Consider the following population, a slight variant from the example presented in Section 3:

\begin{tabular}{|c|c|}
\hline$\pi$ & $\lambda(\pi)$ \\
\hline \hline$e$ & $\frac{1}{2}-2 \varepsilon$ \\
\hline$a_{1} a_{2} a_{4} a_{3} a_{6} a_{5} a_{8} a_{7} a_{10} a_{9}$ & $\frac{1}{5}\left(\frac{1}{2}-\varepsilon\right)$ \\
\hline$a_{2} a_{1} a_{3} a_{4} a_{6} a_{5} a_{8} a_{7} a_{10} a_{9}$ & $\frac{1}{5}\left(\frac{1}{2}-\varepsilon\right)$ \\
\hline$a_{2} a_{1} a_{4} a_{3} a_{5} a_{6} a_{8} a_{7} a_{10} a_{9}$ & $\frac{1}{5}\left(\frac{1}{2}-\varepsilon\right)$ \\
\hline$a_{2} a_{1} a_{4} a_{3} a_{6} a_{5} a_{7} a_{8} a_{10} a_{9}$ & $\frac{1}{5}\left(\frac{1}{2}-\varepsilon\right)$ \\
\hline$a_{2} a_{1} a_{4} a_{3} a_{6} a_{5} a_{8} a_{7} a_{9} a_{10}$ & $\frac{1}{5}\left(\frac{1}{2}-\varepsilon\right)$ \\
\hline$a_{10} a_{9} a_{8} a_{7} a_{6} a_{5} a_{4} a_{3} a_{2} a_{1}$ & $3 \varepsilon$ \\
\hline
\end{tabular}

Using the strategy from the proof above, we can show that $\hat{\pi}=a_{2} a_{1} a_{4} a_{3} a_{6} a_{5} a_{8} a_{7} a_{10} a_{9}$ covers $e$, the ordering consistent with majority preferences. We need to show that (a) $\hat{\pi} T^{\lambda} e$ and (b) for all $\pi^{\prime} \in \Pi, e T^{\lambda} \pi^{\prime} \Rightarrow \hat{\pi} T^{\lambda} \pi^{\prime}$. First we will show that $\hat{\pi} T^{\lambda} e$. For the five orderings in population with weight $\frac{1}{5}\left(\frac{1}{2}-\varepsilon\right)$, we have $f(\pi, \hat{\pi})=1$ and $f(\pi, e)=4$. And, we know $a_{10} a_{9} a_{8} a_{7} a_{6} a_{5} a_{4} a_{3} a_{2} a_{1}$ is closer to $\hat{\pi}$ than $e$, since it is maximally distant from $e$. Thus, $\frac{1}{2}+2 \varepsilon$ of the population is closer to $\hat{\pi}$ than $e$, so $\hat{\pi} T^{\lambda} e$. Now we need to show there cannot exist $\pi^{\prime}$ such that $e T^{\lambda} \pi^{\prime}$ but $\pi^{\prime} T^{\lambda} \hat{\pi}$. Suppose there did exist such a $\pi^{\prime}$. Then, $e T^{\lambda} \pi^{\prime}$ implies that for at least one of the orderings $\pi$ in population other than $e, f(e, \pi) \leq$ $f\left(\pi^{\prime}, \pi\right)$. We know there cannot exist a $\pi^{\prime}, \pi^{\prime} \neq e$, such that $f\left(e, a_{10} a_{9} a_{8} a_{7} a_{6} a_{5} a_{4} a_{3} a_{2} a_{1}\right) \leq$ $f\left(\pi^{\prime}, a_{10} a_{9} a_{8} a_{7} a_{6} a_{5} a_{4} a_{3} a_{2} a_{1}\right)$. So, it must be that this is true for one of the remaining five 
orderings. Since for any of these orderings $f(e, \pi)=4$, we must have $f\left(\pi^{\prime}, \pi\right) \geq 4$ for at least one of those five orderings $\pi$. And, the fact that $\pi^{\prime} T^{\lambda} \hat{\pi}$ implies that we have at least one of the following two cases:

1. For at least one of the orderings with weight $\frac{1}{5}\left(\frac{1}{2}-\varepsilon\right)$, we have $f\left(\pi^{\prime}, \pi\right) \leq f(\hat{\pi}, \pi)$.

2. For both $e$ and $a_{10} a_{9} a_{8} a_{7} a_{6} a_{5} a_{4} a_{3} a_{2} a_{1}$, we have $f\left(\pi^{\prime}, \pi\right)<f(\hat{\pi}, \pi)$.

For case 1 , we know $f(\hat{\pi}, \pi)=1$, so this would imply, $f\left(\pi^{\prime}, \pi\right) \leq 1$ for one of the orderings with weight $\frac{1}{5}\left(\frac{1}{2}-\varepsilon\right)$. This leads to the same violation of the triangle inequality that we reached above, since for any two orderings with weight $\frac{1}{5}\left(\frac{1}{2}-\varepsilon\right)$, we have $f\left(\pi_{i}, \pi_{j}\right) \leq 2$. For case $2, f\left(\pi^{\prime}, e\right)<f(\hat{\pi}, e)$ implies $f\left(\pi^{\prime}, e\right)<5$. And, $f\left(\pi^{\prime}, a_{10} a_{9} a_{8} a_{7} a_{6} a_{5} a_{4} a_{3} a_{2} a_{1}\right)<$ $f\left(\hat{\pi}, a_{10} a_{9} a_{8} a_{7} a_{6} a_{5} a_{4} a_{3} a_{2} a_{1}\right)$ implies $f\left(\pi^{\prime}, a_{10} a_{9} a_{8} a_{7} a_{6} a_{5} a_{4} a_{3} a_{2} a_{1}\right)<40$. But, $f\left(\pi^{\prime}, e\right)<5$ and $f\left(\pi^{\prime}, a_{10} a_{9} a_{8} a_{7} a_{6} a_{5} a_{4} a_{3} a_{2} a_{1}\right)<40$ cannot both hold, since the first implies $\pi^{\prime}$ has fewer than five errors and the second implies it has more than 5 errors. This leads to a contradiction. Thus, order consistency fails for this population.

This example illustrates the difficulty we encounter if we attempt to tighten the sufficiency condition for ordering consistency by imposing a radius around the orderings with common errors. Taking the basic counterexample from above, where the minority orderings all lie relatively close to another, we can move some weight to $a_{10} a_{9} a_{8} a_{7} a_{6} a_{5} a_{4} a_{3} a_{2} a_{1}$ and still arrive at $\hat{\pi}$ covers $e$. Thus, it is not always true that we need the minority orderings to be relatively close to one another in order to have order consistency fail. 


\section{Acknowledgements}

A special thank you to Jerry Green for many helpful conversations about this work. This author also gratefully acknowledges the comments and suggestions of Steven Brams, Lucas Coffman, Drew Fudenberg, Yuichiro Kamada, Klaus Nehring, Alvin Roth, Kenneth Shepsle, Tomasz Strzalecki, William Zwicker, seminar participants at Harvard University, and conference participants at the New York University Graduate Student Political Economy Conference, EconCon 2011, and the Judgment Aggregation and Voting Theory Workshop.

\section{References}

[1] Ahn, D.S. and S. Oliveros. 2012. Combinatorial Voting. Econometrica 80(1), 89-141.

[2] Arrow, K.J. 1951. Social Choice and Individual Values. Wiley, New York.

[3] Baldiga, K.A. and J.R. Green. 2013. Assent-Maximizing Social Choice. Social Choice and Welfare 40(2), pp. 439 - 460.

[4] Banks, J.S. 1985. Sophisticated voting outcomes and agenda control. Social Choice and Welfare 4, pp. 295 - 306.

[5] Besley, T. and S. Coate. 2008. Issue Unbundling via Citizens' Initiatives. Quarterly Journal of Political Science 3(4), pp. 379-397.

[6] Black, D. 1948. On the Rationale of Group Decision Making. Journal of Political Economy, 56 .

[7] Bossert, W. and T. Storcken. 1992. Strategy-proofness of social welfare functions: The use of the Kemeny distance between preference orderings. Social Choice and Welfare, $9,345-360$. 
[8] Bowser, Jennie D. Initiative, Referendum, and Recall. National Conference of State Legislatures. Jennie Drage Bowser. August 2012. < http://www.ncsl.org/legislatureselections/elections/initiative-referendum-and-recall-overview.aspx >

[9] Brandt, F., C. Geist, and P. Harrenstein. Working paper. A note on the McKelvey uncovered set and Pareto optimality.

[10] Chamberlin, J. and P. Courant. 1983. Representative deliberations and representative decisions: Proportional representation and the borda rule. The American Political Science Review, 77(3), pp. 718 - 733.

[11] Copeland, A.H. 1951. A reasonable social welfare function. Unpublished manuscript.

[12] Daniels, H.E. 1969. Round robin tournament scores. Biometrika 56, pp. 295 - 299.

[13] Dasgupta, P. and E. Maskin. 2008. On the Robustness of Majority Rule. Journal of the European Economic Association 6, 5, 949-973.

[14] Felsenthal, D. and M. Machover. 1998. The Measurement of Voting Power: Theory and Practice, Problems, and Paradoxes. Cheltenham, UK, and Northampton, MA, USA: Edward Elgar Publishing Ltd.

[15] Fishburn, P. 1977. Condorcet social choice functions. SIAM Journal of Applied Mathematics 33, pp. 469 - 489.

[16] Fox, J. and K. Shotts. 2013. Delegates or trustees? A theory of political accountability. Working paper.

[17] Frey, B. 1994. Direct Democracy: Politico-Economic Lessons from the Swiss Experience. American Economic Review, 84(2), 338-342.

[18] Gilles, D. 1959. Solutions to general non-zero-sum games in Contributions to the Theory of Games IV. Annals of Mathematics and Statistics, 40, Princeton University Press, Princeton. Edited by A. Tucker and R. Luce. 
[19] Kemeny, J.G. 1959. Mathematics Without Numbers. Daedalus 88. 577-591.

[20] Kemeny, J.G. and J.L. Snell. 1962. Mathematical Models in the Social Sciences, chapter 2, Cambridge: MIT Press.

[21] Laffond, G. and J. Lainé . 2000. Majority voting on orders. Theory and Decision, 49(3), pp. $249-287$.

[22] Laffond, G., J.F. Laslier, and M. Le Bretton. 1993. The bipartisan set of a tournament game, 5(1), pp. 182 - 201.

[23] Lainé , J. A. Ozkes, and M. Sanver. Working paper. Hyper-stable social welfare functions.

[24] Laslier, J.F. 1997. Tournaments Solutions and Majority Voting. Berlin, New York: Springer-Verlag.

[25] Maskin, E. and J. Tirole. 2004. The politician and the judge: accountability in government. American Economic Review, 94(4), 1034-1054.

[26] McGarvey, D.C. 1953. A Theorem on the Construction of Voting Paradoxes. Econometrica $21,608-610$.

[27] McKelvey, R.D. 1986. Covering, Dominance, and Institution-Free Properties of Social Choice. American Journal of Political Science 30(2), 283-314.

[28] Miller, N.R. 1980. A New Solution Set for Tournaments and Majority Voting: Further Graph-Theoretical Approaches to the Theory of Voting. American Journal of Political Science 24(1), 68-96.

[29] Monroe, B. 1995. Fully proportional representation. The American Political Science Review, 89(4), pp. 925 - 940. 
[30] Potthof, R. and S. Brams. 1998. Proportional representation: Broadening the options. Journal of Theoretical Politics, 10(2), pp. 147 - 178.

[31] Schwartz, T. 1972. Rationality and the myth of the maximum. Nous 6, pp. 97 - 117.

[32] Sen, A.K. and P.K. Pattanaik. 1969. Necessary and sufficient conditions for rational choice under majority decision. Journal of Economic Theory 1, 178-202.

[33] Shepsle, K.A. and B.R. Weingast. 1984. Uncovered Sets and Sophisticated Voting Outcomes with Implications for Agenda Institutions. American Journal of Political Science 28(1), 49-74.

[34] Slater, P. 1961. Inconsistencies in a schedule of paired comparisons. Biometrica 48, pp. $303-312$.

[35] Tullock, G. 1967. Towards a Mathematics of Politics. University of Michigan Press. 